\title{
Ion flows in the scrape-off layer with biased limiter: implications for Tore Supra toroidal pumped limiter design
}

\author{
E. Gravier ${ }^{1, a}$, J.P. Gunn ${ }^{1, b}$, J.-L. Lachambre ${ }^{1}$, T. Loarer ${ }^{1}$, \\ G. Mank $^{2, c}$, C. Boucher ${ }^{3}$, K.-H. Finken ${ }^{2, c}$, S. Jachmich ${ }^{4, c}$, \\ M. Lehnen ${ }^{2, \mathrm{c}}$ and G. Van Oost ${ }^{5}$ \\ ${ }^{1}$ Association EURATOM-CEA sur la fusion contrôlée, CEA/DSM/DRFC, CEA-Cadarache \\ F-13108 Saint-Paul-Lez-Durance Cedex, France \\ 2 Institut für Plasmaphysik, Forschungszentrum, Jülich GmbH, EURATOM Association, \\ D-52425 Jülich, Germany \\ ${ }^{3}$ INRS-Energie et matériaux, 1650 boulevard Lionel-Boulet, Varennes, J3X 1S2, Canada \\ ${ }^{4}$ Laboratory for Plasmaphysics, Ecole Royale Militaire-Koninklijke Militaire School, \\ Association 'EURATOM-Belgian State', B-1000 Brussels, Belgium \\ ${ }^{5}$ Department of Applied Physics, Ghent University, B-9000 Gent, Belgium \\ E-mail: Jamie.Gunn@cea.fr
}

Received 30 October 2001, accepted for publication 17 April 2002

Published 6 June 2002

Online at stacks.iop.org/NF/42/653

\begin{abstract}
A simple analytic fluid model based on particle and momentum conservation describes the response of the scrape-off layer to an applied radial electric field. The model explains why the variation of plenum pressure during limiter biasing in TEXTOR shows the opposite behaviour as divertor biasing in Tokamak de Varennes: the mechanical baffles that define the throats of the pump limiter are aligned along the magnetic field, thus preventing collection of the induced perpendicular flux when the drift is directed towards the scoop. Rather, the optimal pumping is expected when the drift is directed away from the scoop due to the increase of the parallel flux needed to satisfy the Bohm-Chodura criterion. Qualitative agreement is obtained with measurements of the parallel flow near the limiter throat, and with measurements of the plenum pressure during negative biasing for both directions of toroidal magnetic field. The results, extrapolated to Tore Supra, predict that the actual design with throats should provide close to optimal pumping with minor modification of the natural radial electric field, but greater performance could be obtained with negative biasing if a toroidally symmetric neutralizer geometry were adopted.
\end{abstract}

PACS numbers: 52.30.-q, 52.25.Xz

\section{Introduction: the CIEL project}

The ultimate performance target of the Tore Supra tokamak is to produce high-power, long-duration discharges ( $25 \mathrm{MW}$ injected to give $1 \mathrm{MW} \mathrm{m}^{-3}$ for $1000 \mathrm{~s}$ ), while actively controlling particle and power balance [1]. All the plasmafacing components were initially designed for long discharges and were actively cooled. However, experience has shown that the uncooled components located far from the plasma receive

\footnotetext{
a Permanent address: Université Henri Poincaré Nancy I, Laboratoire de physique des milieux ionisés et applications, Faculté des Sciences, Bld des Aiguillettes, B.P. 239, 54506 Vandoeuvre-les-Nancy Cédex, France.

b Author to whom correspondence should be addressed.

c Partner in Trilateral Euregio Cluster.
}

radiated power that cannot be neglected on long time scales [2]. After typically $1 \mathrm{~min}$, the effect of outgassing from these areas becomes troublesome, contributing to an increase of the plasma density and to a rise of the impurity content of the plasma core. Obviously, without an active pumping system adapted for longduration discharges, the plasma density cannot be controlled.

An upgrade known as composants internes et limiteur (CIEL) is underway to improve the heat extraction capability of the machine. The first wall consists of actively cooled panels that almost totally cover the vacuum chamber ( $98 \%$ coverage). The principal plasma-facing component is a flat toroidal pump limiter (TPL) located at the bottom of the vessel, covering a surface of approximately $7 \mathrm{~m}^{2}$ (figure 1). It is divided into 12 electrically isolated sectors, each sector being armoured on 
(a)

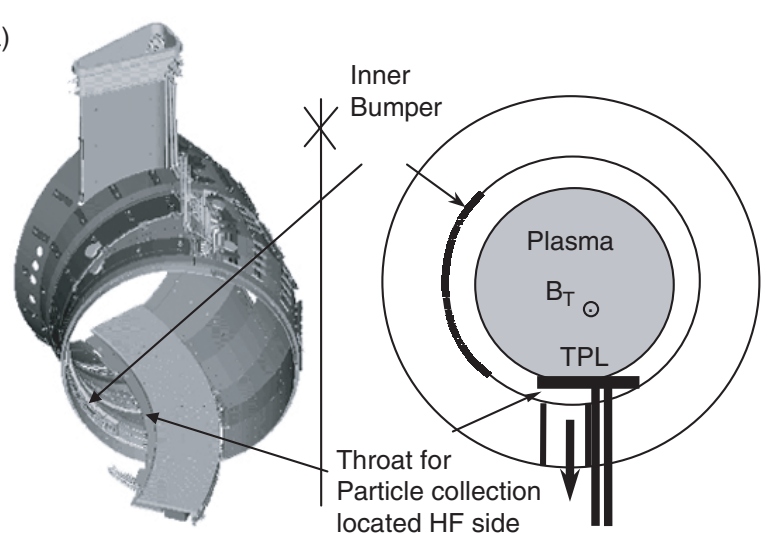

Pumping

(b)

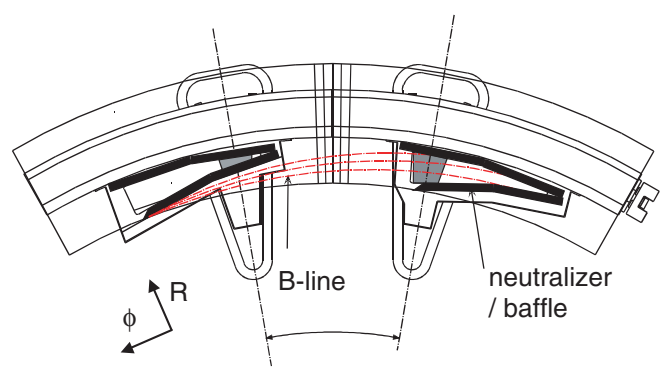

Figure 1. (a) Schematic view of the TPL in Tore Supra in the poloidal section. The pumping ducts are located on the high-field side. (b) Top view of throats, designed for parallel particle flux collection.

the plasma side by 48 actively cooled fingers. Pumping throats are installed underneath each sector on the inboard side. The power exhaust capability of the TPL is $15 \mathrm{MW}$ in steady state. Optimal pumping is expected when the plasma contact point is shifted towards the inboard side, but there is a tradeoff with the increased power deposition on the leading edges of the fingers. Simultaneous control of pumping and impurity generation will be a challenging task, and all possible means of acting on edge particle and power flows must be exploited.

This paper pertains to electrical biasing of the TPL with respect to the first wall. By tailoring the radial electric field in the scrape-off layer (SOL), it should be possible to manipulate the edge flows and poloidal pressure gradients, thereby providing a tool to optimize the pumping independently of the plasma's radial position on the TPL. Preliminary practical considerations of SOL biasing in large tokamaks (modification of SOL flows and pressure gradients, technological requirements, etc) were made by Weynants [3]. Drawing on the long experience of Tokamak de Varennes in the field of divertor biasing [4], we have applied a simple analytic model in a first attempt to identify some of the main physics issues at play. The model is described in section 2. In anticipation of TPL biasing in CIEL, experiments have been performed in the TEXTOR-94 tokamak [5]. Measurements of electric field, parallel flows, and plenum pressure were made during biasing of the ALT-II limiter. The main experimental trends can be explained by the model; the comparison is summarized in section 3 .

\section{Model}

The response of the SOL to imposed electric fields is complex [6]. In a fluid formalism, one finds that the Lorentz force counteracts any other force acting perpendicularly to the magnetic field (electric, pressure gradient, centrifugal, viscous, friction, etc), and gives rise to particle currents perpendicular to both the field and the force directions. Thus in the simplest SOL model without external biasing and uniform magnetic field, one already expects to find plasma parameter asymmetries between the target plates due to the two components of the natural electric field [7] ('natural' signifies any electric field that exists with all plasma-facing components grounded to the first wall): the poloidal presheath electric field gives rise to oppositely directed radial drifts on either side of the stagnation point; the radial electric field, associated with the radial decay of the electron temperature, gives rise to poloidal drifts and pressure gradients [8]. It is not the goal of this paper to contribute to the extensive literature on this topic [9], but merely to isolate and study one aspect of SOL biasing that holds particular relevance for the pumping properties of the TPL, using a simple intuitive model to extract qualitative trends.

The model we use has been described earlier, with slight variations, by other authors, but for clarity we find it useful to show the full derivation of the equations. The approach is inspired by the Mach probe formalism of Hutchinson [10], who modelled the effect of parallel flow on the presheath of a probe, and the model of Lachambre [11] who included poloidal drift effects for the case of divertor biasing. Van Goubergen developed a model to describe ion collection by inclined surfaces in the presence of poloidal drifts $[12,13]$. The results of Van Goubergen's fluid model were fully validated by twodimensional kinetic modelling [14] and experiments with a rotating Mach probe [15] and Gundestrup probe [16-18] in the CASTOR tokamak.

Starting from the three-dimensional ion fluid equations, we make a succession of simplifications until we converge on a set of equations similar to the one Stangeby often employs [19]. The model geometry is a Cartesian slab where the machine coordinates $\left(x_{\phi}, x_{\theta}, x_{\mathrm{r}}\right)$ refer, respectively, to the ignorable toroidal direction in axisymmetric systems, the poloidal direction, and the radial direction (figure 2). The magnetic field lines lie in the $\phi-\theta$ plane, making an angle $\alpha$ with the toroidal direction. A magnetic referential is aligned with the magnetic field where $\left(x_{\|}, x_{\perp}, x_{\mathrm{r}}\right)$ refer, respectively, to the directions parallel to the magnetic field, perpendicular to the magnetic field lying on the magnetic flux surface, and radial across magnetic flux surfaces. We consider the stationary ion continuity equation (neglecting ionization)

$$
\vec{\nabla} \cdot(n \vec{V})=0
$$

and the momentum equation (neglecting collisions) $m \vec{\nabla} \cdot(n \vec{\nabla} \vec{\nabla})=-\vec{\nabla} p-\vec{\nabla}: \stackrel{\leftrightarrow}{\Pi}+\operatorname{Zen}(\vec{E}+\vec{V} \times \vec{B})$,

where $m$ is the ion mass, $n$ is the ion density, $\vec{V}$ is the ion fluid velocity, $p$ is the ion pressure, $\stackrel{\leftrightarrow}{\Pi}$ is the ion stress tensor, $Z$ is the ion charge number, $e$ is the unit charge, $\vec{E}$ is the electric field, and $\vec{B}$ is the magnetic field. The model treats the transport of ion mass uniquely; electrons are not explicitly included, 


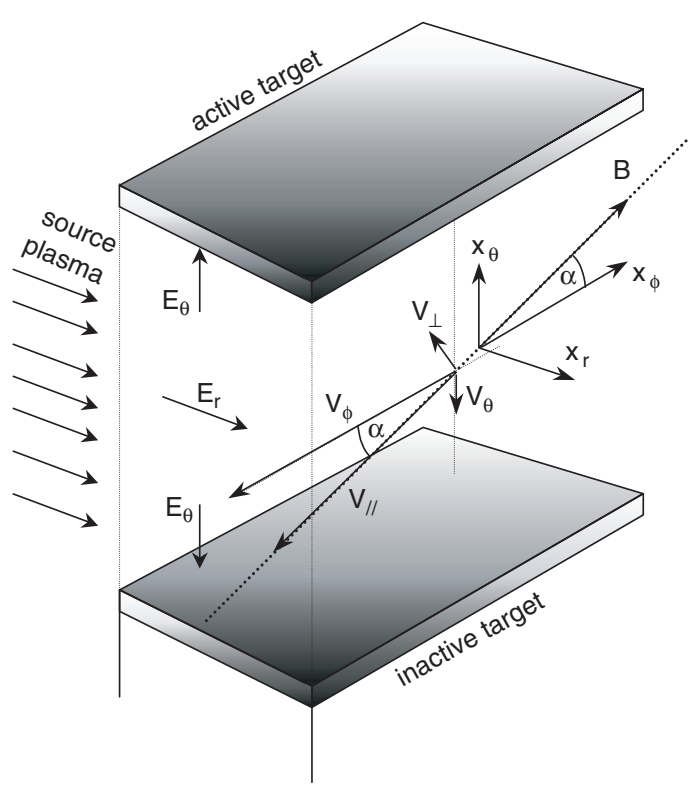

Figure 2. Model geometry. The 'active' target is the one towards which the $E \times B$ drift is directed.

therefore, except for ion and electron temperatures appearing in the following development, we do not use subscripts to distinguish charge species.

The SOL is treated as a slab interfacing via the last closed flux surface (LCFS) to an adjacent slab of uniform source plasma having fixed density $n_{0}$. The transport in the radial direction that connects the two regions is modelled as a purely diffusive, random walk process such that the local radial particle flux is

$$
n V_{\mathrm{r}}=-D_{\mathrm{r}} \frac{\partial n}{\partial x_{\mathrm{r}}}
$$

where $V_{\mathrm{r}}$ is the radial speed and $D_{\mathrm{r}}$ is the anomalous radial diffusion coefficient. The transport is assumed to arise due to turbulent, flute-like eddies over characteristic time and distance scales that are microscopic with respect to those of the SOL. It can be assumed that an individual ion's parallel speed is unaffected since the parallel wave number of the flute-like eddies is much smaller than the perpendicular or radial wave numbers. Hutchinson [10] showed that in such a case, an anomalous shear viscosity term must appear in the stress tensor in order to obtain quantitatively reasonable solutions to the Mach probe problem. However, retaining this term does not significantly modify the main qualitative behaviour of the SOL profiles, and since it prevents us from obtaining an analytic solution, we do not include it in this model.

The radial diffusive particle flux (3) is much larger than the one that is traditionally calculated from the perpendicular component of equation (2):

$$
n V_{\mathrm{r}}=\frac{1}{Z e B}\left(m \vec{\nabla} \cdot\left(n \vec{V} V_{\perp}\right)+\frac{\partial p}{\partial x_{\perp}}-Z e n E_{\perp}\right) .
$$

The inertial contribution (first-term on the right-hand side) is negligible. The second term is the ion diamagnetic drift flux, which must be ignored since we are modelling mass flow rather than electric current [20]. Only the third term need be retained. It is the radial drift driven by the electric field that arises due to the poloidal electron pressure gradient. The ratio of the diffusive flux (3) to the drift flux (4) is then roughly

$$
\frac{D_{\mathrm{r}} B 2 \pi a}{\lambda_{n}\left(k T_{\mathrm{e}} / e\right)} \sim 10,
$$

where $a$ is the tokamak minor radius, $\lambda_{n}$ is the radial density decay length, $k$ is Boltzmann's constant, and $T_{\mathrm{e}}$ is the electron temperature. Typical values for Tore Supra are $D_{\mathrm{r}}=1 \mathrm{~m}^{2} \mathrm{~s}^{-1}$, $B=3.5 \mathrm{~T}, a=0.75 \mathrm{~m}, \lambda_{n}=2 \mathrm{~cm}$, and $T_{\mathrm{e}}=50 \mathrm{eV}$. We note that even though the perpendicular momentum equation is of no use here, it is crucial when modelling electric current flow because the diffusive ion and electron fluxes are ambipolar and thus cancel.

The physical phenomena of interest are dominated by the perpendicular electric drift as seen by writing the radial component of equation (2):

$$
V_{\perp}=\frac{1}{Z e n B}\left(-m \vec{\nabla} \cdot\left(n \vec{V} V_{\mathrm{r}}\right)-\frac{\partial p}{\partial x_{\mathrm{r}}}+\operatorname{Zen} E_{\mathrm{r}}\right) .
$$

Again, it is justified to neglect inertia, and required to ignore the diamagnetic drift, leaving simply

$$
V_{\perp}=\frac{E_{\mathrm{r}}}{B} .
$$

The radial electric field is taken as constant due to the high parallel conductivity of magnetic flux surfaces. Diffusive transport in the perpendicular direction can be ignored unless the angle $\alpha$ is very small, typically less than $1^{\circ}$.

The parallel component of equation (2) is written as

$$
m \vec{\nabla} \cdot\left(n \vec{V} V_{\|}\right)=-\frac{\partial p}{\partial x_{\|}}+Z e n E_{\|},
$$

where the viscosity has been dropped. We assume isothermal Maxwellian electrons with temperature $T_{\mathrm{e}}$ and replace the electric field by the Boltzmann relation

$$
E_{\|}=-\frac{k T_{\mathrm{e}}}{e n} \frac{\partial n}{\partial x_{\|}} .
$$

Ion and electron charge densities are supposed to be equal. An adiabatic equation of state is assumed for the parallel ion motion:

$$
p=n k T_{\mathrm{i}} \propto n^{\gamma},
$$

where $T_{\mathrm{i}}$ is the ion temperature and $\gamma$ is the isentropic exponent, allowing us to write

$$
\frac{1}{p} \frac{\partial p}{\partial x_{\|}}=\gamma \frac{1}{n} \frac{\partial n}{\partial x_{\|}} .
$$

Inserting the cross-field flux (3), and separating out the radial divergences, equations (1) and (8) become

$$
\begin{gathered}
\frac{\partial}{\partial x_{\|}} n V_{\|}+\frac{\partial}{\partial x_{\perp}} n V_{\perp}=D_{\mathrm{r}} \frac{\partial^{2}}{\partial x_{\mathrm{r}}^{2}} n, \\
\frac{\partial}{\partial x_{\|}} n\left(V_{\|}^{2}+c_{\mathrm{s}}^{2}\right)+V_{\perp} \frac{\partial}{\partial x_{\perp}} n V_{\|}=D_{\mathrm{r}} \frac{\partial}{\partial x_{\mathrm{r}}} V_{\|} \frac{\partial n}{\partial x_{\mathrm{r}}},
\end{gathered}
$$

where $V_{\perp}$ is taken as constant as discussed above and the ion sound speed is defined as

$$
c_{\mathrm{s}}^{2} \equiv \frac{k\left(Z T_{\mathrm{e}}+\gamma T_{\mathrm{i}}\right)}{m_{\mathrm{i}}} .
$$


Following Hutchinson [10], we assume that the radial divergence of a quantity $\psi$ can be approximated as its difference between the source region and the SOL, divided by the characteristic width $\lambda$ of the SOL:

$$
\begin{aligned}
& \frac{\partial \psi}{\partial x_{\mathrm{r}}} \approx-\frac{\psi_{0}-\psi}{\lambda}, \\
& \frac{\partial^{2} \psi}{\partial x_{\mathrm{r}}^{2}} \approx \frac{\psi_{0}-\psi}{\lambda^{2}},
\end{aligned}
$$

yielding

$$
\begin{gathered}
\frac{\partial}{\partial x_{\|}} n V_{\|}+\frac{\partial}{\partial x_{\perp}} n V_{\perp}=\frac{D_{\mathrm{r}}}{\lambda^{2}}\left(n_{0}-n\right), \\
\frac{\partial}{\partial x_{\|}} n\left(V_{\|}^{2}+c_{\mathrm{s}}^{2}\right)+V_{\perp} \frac{\partial}{\partial x_{\perp}} n V_{\|}=0,
\end{gathered}
$$

where we have supposed that the source plasma is stagnant $\left(V_{\|, 0}=0\right)$. The equations are rotated into the machine referential:

$$
\begin{gathered}
\frac{\mathrm{d}}{\mathrm{d} x_{\theta}} n V_{\theta}=\frac{D_{\mathrm{r}}}{\lambda^{2}}\left(n_{0}-n\right), \\
\frac{\mathrm{d}}{\mathrm{d} x_{\theta}} n\left[V_{\theta}\left(V_{\theta}-V_{\perp} \cos \alpha\right)+c_{\mathrm{s}}^{2} \sin ^{2} \alpha\right]=0 .
\end{gathered}
$$

The speeds are related by

$$
V_{\theta}=V_{\|} \sin \alpha+V_{\perp} \cos \alpha .
$$

Now we introduce a normalization. A frequency is defined that describes the exchange rate of particles between the source plasma and the SOL:

$$
W \equiv \frac{D_{\mathrm{r}}}{\lambda^{2}}
$$

Speed is normalized by the poloidal ion sound speed $c_{\mathrm{s}} \sin \alpha$, density by the source density $n_{0}$, and distance by $c_{\mathrm{s}} / W$ so that the equations become

$$
\begin{gathered}
\frac{\mathrm{d}}{\mathrm{d} x_{\theta}} n M_{\theta}=1-n, \\
\frac{\mathrm{d}}{\mathrm{d} x_{\theta}} n\left[M_{\theta}\left(M_{\theta}-M_{\perp}\right)+1\right]=0,
\end{gathered}
$$

where we define the poloidal Mach number

$$
M_{\theta} \equiv \frac{V_{\theta}}{c_{\mathrm{s}} \sin \alpha}
$$

and the perpendicular Mach number

$$
M_{\perp} \equiv \frac{V_{\perp}}{c_{\mathrm{S}} \tan \alpha} .
$$

Coupled ordinary differential equations are obtained for the density and the poloidal Mach number:

$$
\begin{gathered}
\frac{\mathrm{d} n}{\mathrm{~d} x_{\theta}}=\frac{\left(2 M_{\theta}-M_{\perp}\right)(1-n)}{M_{\theta}^{2}-1}, \\
\frac{\mathrm{d} M_{\theta}}{\mathrm{d} x_{\theta}}=-\frac{\left(M_{\theta}^{2}-M_{\theta} M_{\perp}+1\right)(1-n)}{n\left(M_{\theta}^{2}-1\right)} .
\end{gathered}
$$

These are exactly the same form as Hutchinson's equations (8) and (9) in [24], with the viscosity coefficient set to zero, with the perpendicular Mach number replacing the external flow Mach number in the connected Mach probe problem, and with the projected poloidal velocity replacing the purely parallel velocity. As derived by Van Goubergen [12], the singularity in the denominator gives the Bohm-Chodura boundary condition $\left(M_{\theta}= \pm 1\right)$ [21]. Hutchinson also derived it, but by means of a Galilean transformation [22]. We assume it occurs at the plasma-sheath interface in front of the targets. This choice is not arbitrary, but is recommended by Cohen and Ruytov [23], who demonstrated that sonic transitions occur at 'effective nozzles' (points where the source terms become zero, such as target plates, magnetic pinches, or neutral cushions), and that these transitions must come naturally from the fluid equations. We note, however, that this criterion arises from the stationary equations; other solutions might exist in a dynamic model in which a stable, but time-varying sheath and presheath exist. In our notation the Bohm-Chodura criterion is written as

$$
M_{\theta}=M_{\|}+M_{\perp}= \pm 1,
$$

where the parallel Mach number is defined as

$$
M_{\|}=\frac{V_{\|}}{c_{\mathrm{s}}} .
$$

These Mach numbers refer to the poloidal projection of each velocity component. Formulated this way, equation (29) reflects the fact that a small perpendicular drift results in huge changes of the parallel flow (for the usual case of small pitch angle $\alpha$ of the magnetic field lines with respect to the toroidal direction).

Dividing equation (27) by equation (28) we obtain the well known equation for the density

$$
n=\frac{K_{1}}{M_{\theta}^{2}-M_{\theta} M_{\perp}+1},
$$

where $K_{1}$ is a constant of integration. Hutchinson [24] substituted the density into equation (28) to obtain an analytic solution for the Mach number as a function of poloidal distance. The difference between our and Hutchinson's solution is that he imposes the SOL density and finds the corresponding system length that satisfies particle flux balance, whereas we impose the system length and treat the SOL density as an eigenvalue. By doing the latter, the constants of integration become embedded in a transcendental equation that must be solved numerically. We follow the example of Stangeby [19] and set the source term on the right-hand side of equation (23) to a constant instead of $1-n$ in order to obtain a solution that is easy to manipulate:

$$
\frac{2 K_{1} M_{\theta}}{M_{\theta}^{2}-M_{\theta} M_{\perp}+1}=x_{\theta}+K_{2} .
$$

We choose the value 0.5 for the source term, since we know from the numerical solution of the exact equations that the stagnation density is roughly half the source density $n_{0}$ for the zero biasing case. Mathematically, the consequences are not too severe; the overall shape of the solution resembles the one obtained using the correct source term. In any case, even 
the numerical solution is quite approximate due to the neglect of shear viscosity [25]; all these points will be adressed in another paper where we compare with a kinetic model. For now we admit the increasingly qualitative nature of the results and forge ahead.

Applying the boundary conditions

$$
\left.M_{\theta}\right|_{x=-1 / 2}=-1
$$

and

$$
\left.M_{\theta}\right|_{x=+1 / 2}=+1,
$$

we get the solution

$$
\begin{gathered}
n=\frac{1}{2} \frac{1-(1 / 4) M_{\perp}^{2}}{M_{\theta}^{2}-M_{\theta} M_{\perp}+1}, \\
\frac{\left(1-(1 / 4) M_{\perp}^{2}\right) M_{\theta}}{M_{\theta}^{2}-M_{\theta} M_{\perp}+1}=x_{\theta}+\frac{1}{4} M_{\perp} .
\end{gathered}
$$

Immediately one can define the location of the poloidal stagnation point where $M_{\theta}=0$ :

$$
x_{\theta 0}=-\frac{1}{4} M_{\perp} .
$$

Equation (36) is inverted to give poloidal Mach number as a function of position:

$$
M_{\theta}=\frac{1+M_{\perp} x_{\theta}-\sqrt{1+x_{\theta}^{2}\left(M_{\perp}^{2}-4\right)-(1 / 4) M_{\perp}^{2}}}{2\left(x_{\theta}+(1 / 4) M_{\perp}\right)} .
$$

Henceforth, we adopt the terminology used at TdeV [4]: the target towards which the $E \times B$ drift is directed $\left(M_{\perp}>0\right)$ is called the 'active' target, while the other one $\left(M_{\perp}<0\right)$ is 'inactive'. The model results are shown in figure 3 for three values of $M_{\perp}$. Without biasing $\left(M_{\perp}=0\right)$, all quantities are symmetric about $x_{\theta}=0$. The effect of biasing is to push the density towards the active target, and the poloidal stagnation point towards the inactive target [26]. Biasing constitutes a form of 'momentum injection' into the SOL: one sees that even though the Mach number at each target is pinned to the poloidal sound speed, the poloidally averaged Mach number increases proportionally to bias (as was first shown by Tendler [7]), and the poloidal flux at each target varies linearly with bias. For the case of isothermal ions, the ratio of the target densities is equal to the ratio of the pressures. One can show that the pressure ratio is equal to the ratio of the distances from each target to the poloidal stagnation point:

$$
\frac{n_{\text {active }}}{n_{\text {inactive }}}=\frac{+1 / 2-x_{\theta 0}}{x_{\theta 0}-(-1 / 2)}=\frac{2+M_{\perp}}{2-M_{\perp}} .
$$

Thus, we recover the qualitative result that explains the behaviour of axisymmetric divertors during unipolar biasing [8].

The most striking effect is on the parallel speed and parallel flux. As dictated by the Bohm-Chodura criterion, the parallel speed increases at the inactive target and decreases at the active target to balance the perpendicular drift. The parallel flow opposes the pressure gradient built up by the Lorentz force in the SOL. For large $M_{\perp}>1$, the parallel speed at the active target even reverses, implying that situations may exist where the parallel flow has the same sign everywhere

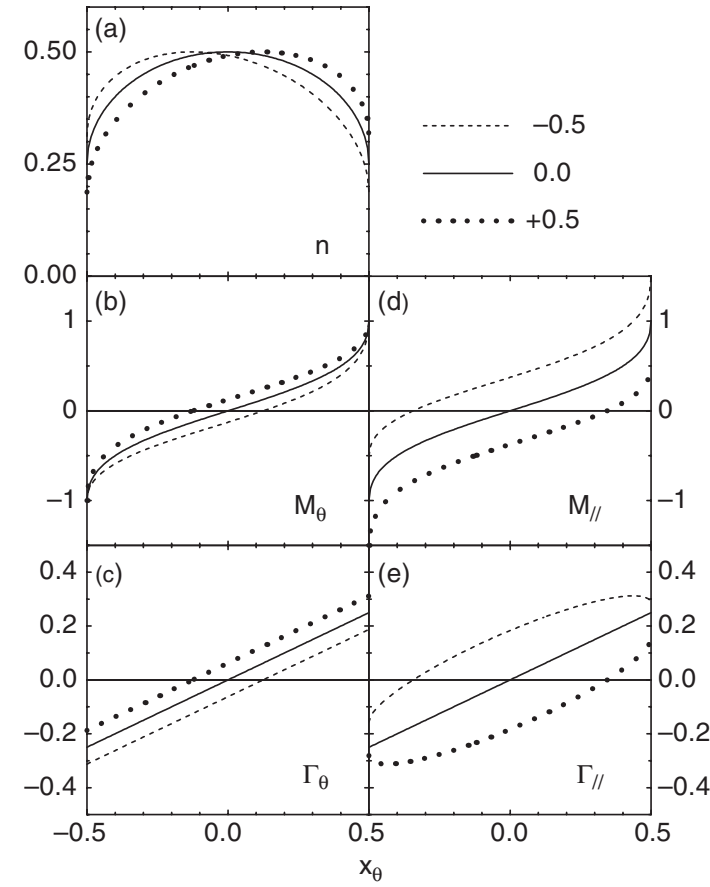

Figure 3. Poloidal profiles of $(a)$ density, $(b)$ poloidal Mach number, $(c)$ poloidal flux, $(d)$ parallel Mach number, and $(e)$ parallel flux. Three values of bias are shown. For $M_{\perp}=-0.5$ (- - - ) the target at $x_{\theta}=-0.5$ is active. For $M_{\perp}=0$ ( $(-)$ the profiles are symmetric about $x_{\theta}=0$. For $M_{\perp}=+0.5(\cdots \cdots)$ the target at $x_{\theta}=+0.5$ is active. The important message in this figure is that even though the density decreases at the inactive target, the parallel flux increases to satisfy the Bohm-Chodura criterion.

in the SOL. Prudence is therefore required when interpreting measurements of parallel Mach number: parallel flow reversal does not necessarily imply poloidal flow reversal out of an axisymmetric divertor into the SOL! In general, the fluid flow does not follow field lines, but arrives at the active target with a large angle (almost purely poloidal flow) for perpendicular Mach number roughly unity, becoming almost purely toroidal for larger drift speeds due to the reversed, supersonic parallel flow. At the inactive target the flow is always at angles between parallel and purely toroidal. The TPL pumping throats in Tore Supra, as in TEXTOR, are equipped with mechanical baffles aligned nearly parallel to the magnetic field, and therefore obstruct the perpendicular component of the flow. At the active throat, therefore, one expects most of the recycling to occur on the back sides of the baffles, and a reduction of plenum pressure (figure 4).

The target plasma parameters calculated with the analytic model are plotted against bias in figure 5. To anticipate the real behaviour of the TPL during biasing, we assume it only collects parallel flux. From equation (35) with $M_{\theta}=+1$ and $M_{\|}=1-M_{\perp}$ the parallel flux at the target is written:

$$
\Gamma_{\|, \text {target }}=\frac{1}{8}\left(2+M_{\perp}\right)\left(1-M_{\perp}\right) .
$$

This expression is plotted in figure $5(c)$ where we see that the parallel flux varies non-linearly with bias, and responds asymmetrically with reversed bias polarity. At the active target, the parallel flux plummets rapidly below zero at the expense of the increasing perpendicular $E \times B$ flux, while at the inactive 
(a)

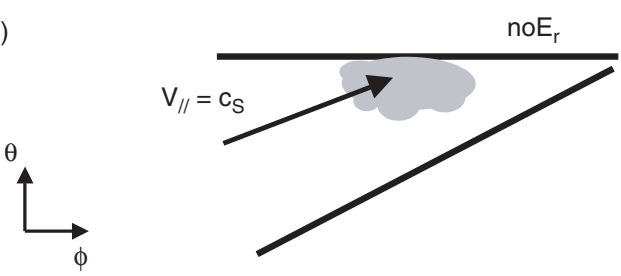

(b)

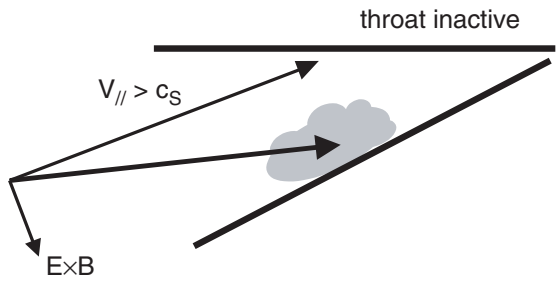

(c)

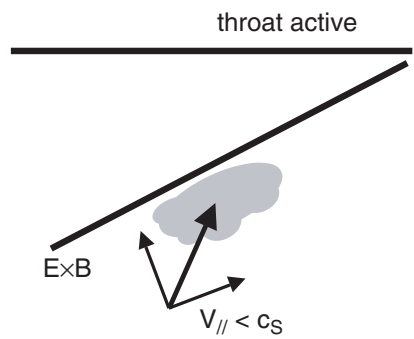

Figure 4. Schematic of ion flow into the V-shaped throat of the Tore Supra TPL. The gray clouds represent the location of maximum recycling. (a) In the case of no radial electric field the parallel flow is equal to the sound speed. $(b)$ The parallel speed increases if the $E \times B$ drift is directed away from the scoop, but the total flow is still collected. (c) If the $E \times B$ drift is towards the scoop, the parallel speed drops and the total flow vector can be such that the flow does not enter the scoop at all.

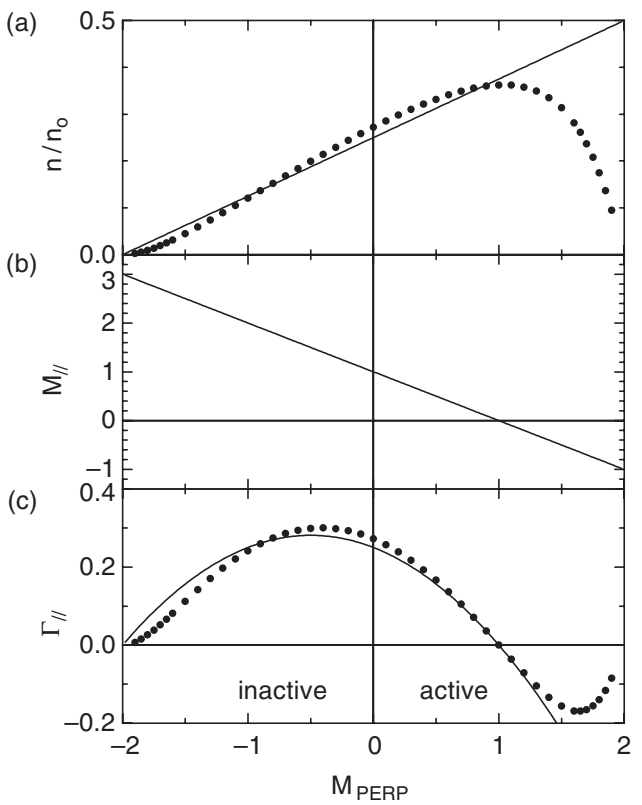

Figure 5. Target plate $(a)$ density, $(b)$ parallel Mach number, and (c) parallel flux as a function of bias. Solid curves are the analytic model with constant source term, and the dots are the numerical solution of Hutchinson's zero-viscosity equations. The target is active (inactive) for positive (negative) values of perpendicular Mach number. target, it increases somewhat to a maximum at $M_{\perp}=-0.5$ before dropping again at higher values of bias. Negative parallel flux does not imply plasma flowing out of the throats! It merely indicates that the flow has such a large angle that no plasma whatsoever can be pumped. The numerical solution of equations (27) and (28) is superimposed for comparison. This is the inviscid Mach probe model described by Hutchinson [24]. His analytic solution assumes that the external parallel flow speed is always less than sonic such that one can integrate (using our notation) from $M_{\theta}=M_{\perp}$ to $M_{\theta}= \pm 1$. In fact, well-behaved solutions exist for $M_{\perp}<2$, and therefore the value $M_{\theta}=M_{\perp}$ does not necessarily exist in the SOL. Instead of using his analytic solution, we integrate numerically from the stagnation point to $M_{\theta}= \pm 1$. The stagnation point density is an eigenvalue, and must be chosen such that the singularities occur at the target plates. The two solutions are in relatively good agreement except for $M_{\perp}>1$. The analytic model breaks down at these values because the choice of source strength is no longer appropriate.

It appears that even though the density decreases at the inactive target, the increase of the parallel flow needed to satisfy the Bohm-Chodura criterion is enough to compensate over quite a broad bias window. The optimal pumping in the TPL should occur when the throat is inactive, exactly the opposite to the axisymmetric divertor. The increase in plenum pressure is expected to be modest (of the order of 10-20\%) compared to the zero radial electric field case. Depending on the magnetic field direction, the positive natural electric field could be such that the target is active without biasing, in which case one would need to cancel or even reverse the radial electric field, leading, as will be shown below, to improvements of the order of 50\%. If one could eliminate the baffles and adopt a toroidally symmetric target geometry, one could reasonably hope to recover the amazing performance obtained in TdeV where pressure increases of $500 \%$ were obtained at the active target [27]. The flexibility that would be gained by an open geometry must of course be weighed against the possible loss of pumping efficiency. Simulations using the EIRENE neutral transport code (without biasing effects) showed that the pumping efficiency would drop by at least a factor of 5 if the V-shaped baffles were removed [28]. Perhaps by placing a toroidally symmetric, grounded return electrode near the throat but at a slightly larger minor radius than the biased neutralizer, the pumping efficiency could be maintained, at the same time as providing access to the positive biasing regime.

\section{Limiter biasing in TEXTOR}

The ALT-II belt limiter in the TEXTOR tokamak is biasable [29], therefore, providing an opportunity to test the desired result of improved pumping by means of unipolar biasing. The experiments were performed with plasma current $I_{\mathrm{p}}=$ $\pm 350 \mathrm{kA}$, and toroidal magnetic field $B_{\mathrm{tor}}= \pm 2.25 \mathrm{~T}$ (giving an edge safety factor $q_{\text {edge }}=3.9$ ), with the toroidal belt limiter at a fixed radius of $r=a=46 \mathrm{~cm}$, neutral beam (co-injection) heating of $1.4 \mathrm{MW}$, and a plasma density of $n_{\mathrm{e}}=2.8 \times 10^{19} \mathrm{~m}^{-3}$. The general properties of the induced radial electric field are described in section 3.1. The model prediction is compared with the measured parallel Mach number in section 3.2, and with the measured plenum pressure in section 3.3 . 


\subsection{Radial electric field}

The decision to make the TPL biasable was partly inspired by the spectacular results obtained with divertor biasing in Tokamak de Varennes [4]. However, there are differences between limiter tokamaks and divertor tokamaks, and one is obliged to ask if similar results can be expected in Tore Supra. The principal difference is with the geometry of the biased components. In a divertor, the target plates are biased with respect to the vacuum vessel, and the radial electric field is established in the vicinity of the magnetic flux surfaces that electrically connect the target to the grounded return electrode, usually another target plate or baffle situated inside the divertor. The current flows along field lines from the target, radially across flux surfaces, and is finally returned to the wall. A radial electric field can be established for either bias polarity. In contrast, the entire mechanical structures of both the ALT-II limiter in TEXTOR-94 and the TPL in Tore Supra are biased, so the voltage is applied from the separatrix all the way to the vacuum vessel wall. The return electrode is in fact the inboard bumper limiter, situated between the two sides of the pump limiter. A part of the current is thus returned along the field lines between the limiter and the bumper. It is nearly impossible to set up a radial electric field with positive biasing because the applied voltage is dropped across the sheath of the bumper, as illustrated in figure 6(a). Biasing is therefore limited to negative polarity (figure $6(b)$ ). Another difficulty is that the electric field is rather far from the separatrix in a limiter experiment, typically one or more density decay lengths. This is due to the requirement that the bumper be located further from the separatrix than the pumping throats, in order to avoid shadowing them. Nonetheless, measurements in TEXTOR-94 show that electric fields can be set up that have similar strengths as those seen in divertor SOLs. Figure 7
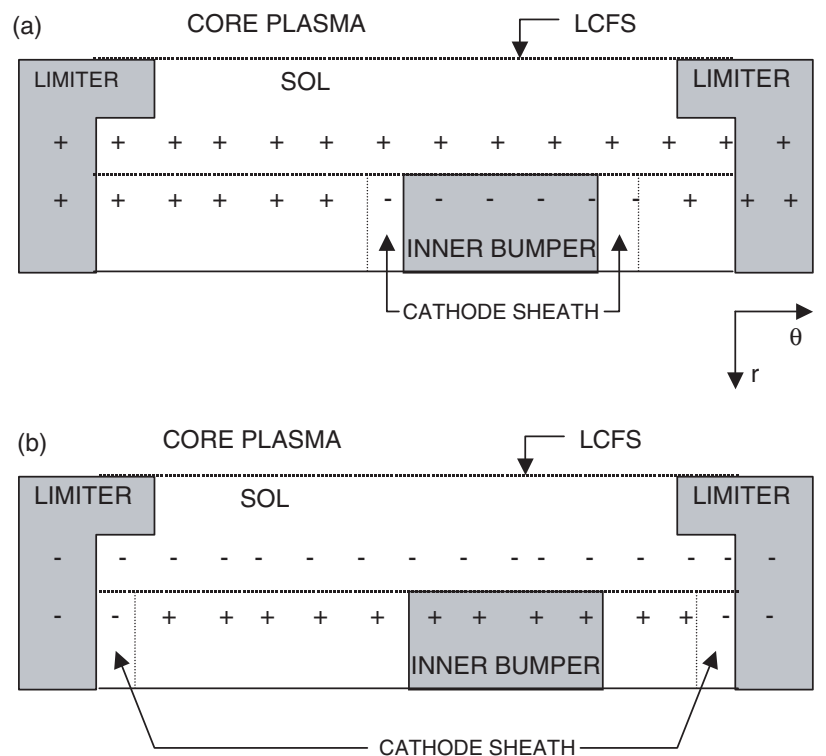

Figure 6. Schematic of two-dimensional spatial distribution of electric potential in a biased SOL for $(a)$ positive biasing and (b) negative biasing. The potential drop on a given field line is almost entirely accomodated by the sheath of the cathode. In positive biasing the inner bumper limiter is the cathode, and so a radial electric field is almost impossible to establish in the SOL. shows the electric field in the SOL of TEXTOR-94 at two radii as a function of the negative applied voltage. $E_{\mathrm{r}}$ is deduced from the plasma potential profile measured by a probe on the outboard midplane. These measurements were taken close to the radial position of the bumper, corresponding, as expected, to the maximum radial electric field. It is seen that a rather moderate applied voltage of $-75 \mathrm{~V}$, corresponding roughly to two or three times the edge $T_{\mathrm{e}}$, is enough to equilibrate the natural electric field which was around $+2 \mathrm{kV} \mathrm{m}^{-1}$ in these experiments. The influence of various limiters and antenna structures on local measurements was clearly shown by detailed connection analysis in [30]. In those experiments, a probe located on top of the machine between two grounded limiters measured strong local radial electric fields behind the limiter radius for both bias polarities, whereas a probe located at the outboard midplane, connected on one side to ALT-II and on the other to the bumper, revealed that no electric field was created during positive biasing (figure 8). The reciprocating probe measurements reported in figure 2 of [29] clearly show the establishment of a strong radial electric field at the same radial position for both bias polarities. The profile during positive biasing resembles the one that we measured on top of the machine between the two grounded limiters (Gundestrup probe measurements in figure 8), therefore those old measurements were also taken in a similar geometry, since, as we have shown, it is impossible to build up a radial electric field on field lines connected to the biased belt limiter.

In the first experiments of biasing on ALT-II [29], Langmuir probes were installed in scoops on both sides of the limiter. The data presented are sparse and only for extreme values of bias voltage (the useful regime is biasing voltages of the order of a few edge electron temperatures), but nonetheless, the results are coherent with our model. In figure 8 of [29], measurements of ion flux inside the scoops were shown for three values of bias voltage. For positive bias, there is no change of the flux. This makes sense because no radial electric field is generated in front of the scoops. During

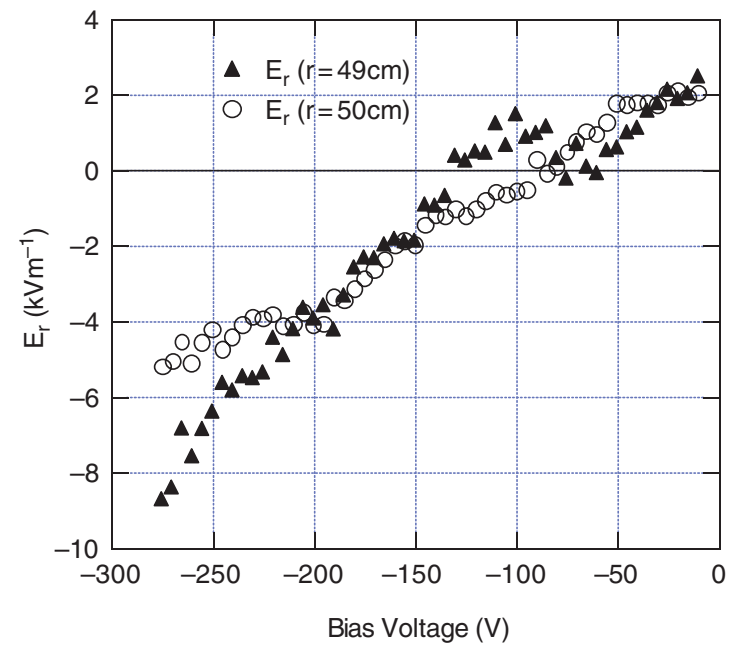

Figure 7. Radial electric field measured by a Gundestrup probe at the outer midplane as a function of limiter bias voltage. The measurements were taken at two radial positions near the flux surface that connects the bumper to ALT-II, and where the radial electric field maximum occurs. The natural radial electric field is cancelled for modest voltages around $-75 \mathrm{~V}$. 


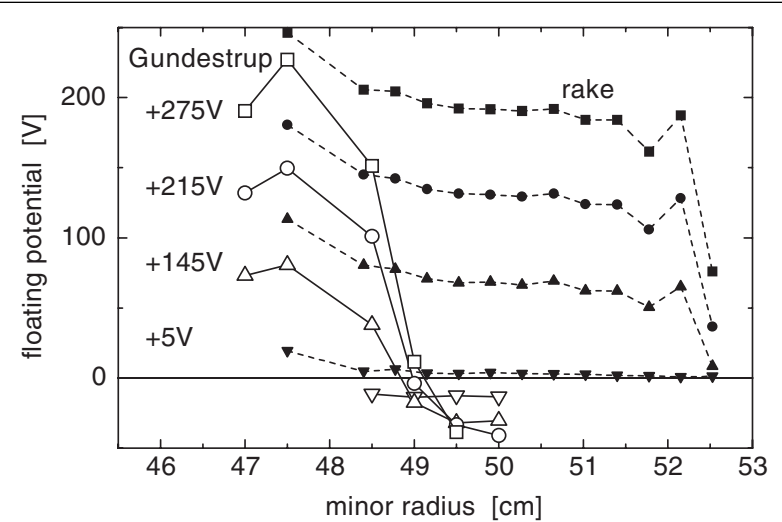

Figure 8. Radial profiles of floating potential for four values of positive limiter biasing. The open symbols are Gundestrup measurements on top of the torus, between two grounded electrodes (the inner bumper and the upper poloidal limiter). The steep gradients occur at the radial position where the probe moves from the limiter shadow onto biased field lines. The solid symbols are rake probe measurements on the outboard midplane, connected to the positive ALT-II limiter on one side, and the grounded inner bumper on the other. The radial electric field is unmodified by positive biasing, except of course very close to the liner (where there is practically no plasma)

negative biasing, the flux into the ion side (inactive scoop with $M_{\perp}<-0.5$ ) decreases somewhat due to the low plasma pressure. The flux into the electron side (active scoop with positive $M_{\perp}$ ) disappears completely because the parallel flux is zero or even reversed, and the ions cannot get into the throat. These results can be simply explained by our model (figure $5(c)$ ), providing a plausible alternative to changes of the radial decay length. In reality, both radial and poloidal flows must be considered together, so one explanation does not preclude the other.

\subsection{Parallel Mach number}

The particle collection in TEXTOR-94 and Tore Supra is exclusively limited to the parallel component of the total outflux because the scoop walls that are oriented parallel to the field lines obstruct the poloidal $E_{r} \times B$ flow. The parallel flow is strongly modified by the imposed radial electric field. To first order, the parallel flow everywhere in the SOL has a tendency to adjust in order to balance the perpendicular drift. The pitch angle of the magnetic field lines is small in tokamaks (typically $5^{\circ}$ ), so the effect on the parallel flow is important. A good test of the model, therefore, is to measure the change of the parallel flow provoked by limiter biasing. A Gundestrup probe [31] was positioned at the outboard midplane, just above the ALT-II limiter, during experiments of negative biasing with reversed magnetic field and plasma current. Without biasing, a near-sonic parallel flow directed towards the low-field side of the limiter was recorded. As the bias voltage was ramped down, the parallel flow dropped sharply, almost to stagnation, in response to the increasing $E_{r} \times B$ drift, directed towards the low-field side of the limiter. The measured parallel Mach number is plotted against the measured perpendicular Mach number (derived from the local radial electric field of figure 7) in figure 9. In order to make this comparison, we are obliged to guess a value for the ion

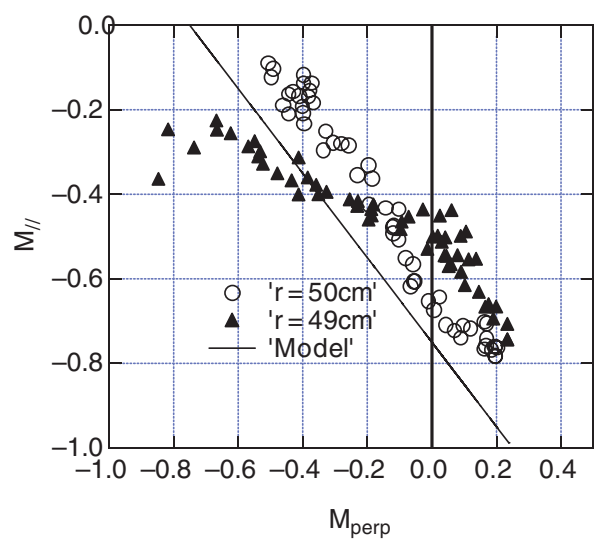

Figure 9. Parallel Mach number at the same radial locations as figure 6, with perpendicular Mach number calculated from the radial electric field data. The Gundestrup probe is at the outboard midplane, just above the ALT-II limiter (situated $45^{\circ}$ below the midplane). The $E \times B$ drift is downward at the probe location toward the active low field side throats. The parallel Mach number decreases as the drift magnitude increases, in order to maintain the poloidal flow roughly constant, in accordance with the Bohm-Chodura criterion.

sound speed because the ion temperature is unknown. Taking the reasonable value of $c_{\mathrm{s}}=\sqrt{(1+5 / 3) T_{\mathrm{e}} / m_{\mathrm{i}}}$, for example, we can obtain good quantitative agreement between the model and the measurement, with $T_{\mathrm{e}}=30 \mathrm{eV}$, as measured by the Gundestrup probe.

The simple analytic model is validated by experiment, lending confidence to the interpretation of pressure measurements in the next section. From the standpoint of plasma engineering, it is significant that these effects are observed at the radial position of the interface between biased and grounded flux surfaces. In TEXTOR, the throat entrance is between $r=48$ and $50 \mathrm{~cm}$, and the bumper is at roughly $49.5 \mathrm{~cm}$, implying that the particle fluxes are modified at a useful radial position in order to be pumped. The important role of the return electrode is clear. By acting on the plasma position and/or the radial distance between the TPL and the bumper (or other grounded limiters or RF antennae) in Tore Supra, it will be possible to guide the induced parallel flux into the plenum.

\subsection{Plenum pressure}

A series of experiments have been performed on TEXTOR94 for positive and negative biasing with normal and reversed $I_{\mathrm{p}}-B_{\mathrm{T}}$ directions $[5,30]$. The parallel particle flux is strongly modified by an imposed radial electric field, and if the flux can be guided into the scoops, there should be changes of the plenum pressure. As explained above, only negative biasing leads to the establishment of significant radial electric fields, so we do not consider the positive biasing results here. The measured pressures are plotted against the measured perpendicular Mach number for both field directions in figure 10. The pressures are normalized to fit onto the model results for the parallel flux into the high-field side scoop. If the pumping were symmetrical, the pressure would be maximum for zero perpendicular Mach number, when the applied bias cancels the natural radial electric field, but the highest pressures 


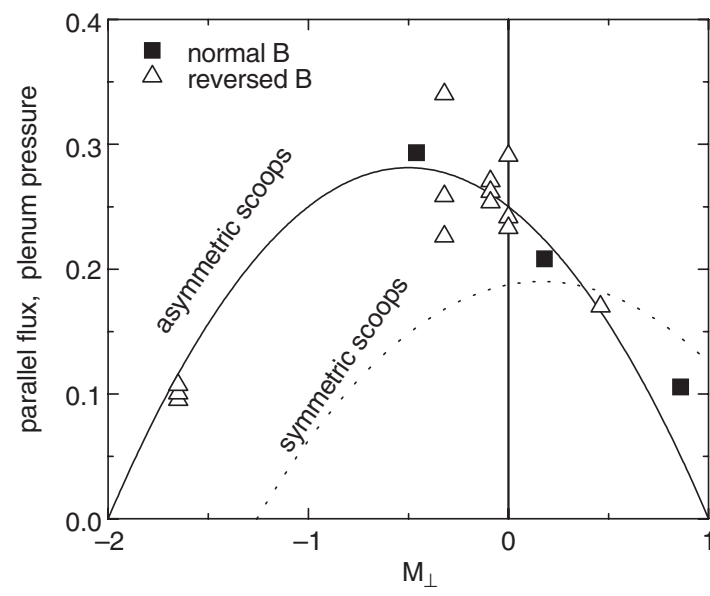

Figure 10. Normalized plenum pressures beneath different blades of the ALT-II limiter as a function of bias, for normal $B-I \mathrm{p}$ direction $(\square)$ and reversed $B-I \mathrm{p}$ direction $(\triangle)$. The measurements are normalized to the model result for the collected parallel flux, assuming that only the high-field side scoops collect. The dotted curve shows the expected symmetric result if both scoops collect with the same efficiency. The best pumping is obtained when the HFS scoops are inactive.

are obtained when the poloidal drift is directed away from the high-field side scoop. This implies that the high-field side scoops collect plasma more efficiently than the lowfield side scoops, within the context of our physical model. The uncertainty of the plasma position in TEXTOR-94 is at least $1 \mathrm{~cm}$, comparable to the density decay length, so it is possible that the plasma was not centred on ALT-II during these experiments. To fit our model prediction, the plasma would have to be leaning on the high-field side edge of the limiter so that in radial magnetic coordinates the highfield side scoops are closer to the LCFS than the low-field side scoops. The interpretation of the data is obviously disputable given the scatter, but in any case, based on our simple model, in agreement with sophisticated numerical modelling by Gerhauser et al using the TECXY code [32], the pumping will always be optimized by the application of radial electric fields of the order required to cancel the natural one in symmetric pumping systems, or to reverse it in asymmetric systems. The implications for Tore Supra-CIEL are encouraging. Since throats are only located on the highfield side of the TPL, an improvement of the particle collection of about $50 \%$ is foreseen for negative biasing in a reversed $I_{\mathrm{p}}-B_{\mathrm{T}}$ configuration. Plasma plugging at higher core densities might help to amplify this improvement.

\section{Conclusions}

The experimental results from TEXTOR-94 show that biasing creates a strong electric field in the SOL and that both the parallel and poloidal particle flux are significantly modified. Simple one-dimensional fluid modelling has illustrated the different ion collection mechanisms operating in poloidal divertors and pump limiters. The poloidal flux that leads to pressure increase at the active target in divertors cannot be collected by a pump limiter because the throat walls obstruct the flow. The optimal pumping is expected when the throat is inactive, due to the increase of the parallel flux needed to satisfy the Bohm-Chodura criterion. Moderate bias voltages of the order of a few electron temperatures are required. Only negative biasing is useful due to the shorting effect of the cathode sheath. To make use of positive biasing, a grounded return electrode would have to be placed close to the limiter throat. In Tore Supra, to have the (inboard) throats inactive in negative biasing requires reversal of the toroidal field and plasma current. In that case a 50\% improvement of pumping is expected due to the reversal of the natural radial electric field. On the other hand, good performance could be obtained with normal field direction and negative biasing (active throats) if the $\mathrm{V}$-shaped throat walls were discarded in favour of a toroidally symmetric neutralizer. A study of alternate baffle geometries is warranted in order to avoid the degraded pumping efficiency that would be expected in a completely open geometry.

\section{Acknowledgments}

The Cadarache authors are grateful to the TEC Directorate for having made this series of experiments possible, and to their TEXTOR co-authors for their lovely hospitality. J.P. Gunn acknowledges V. Fuchs for helpful discussions. All the authors warmly thank Jean-Yves Pascal, R. Komen, A. Hiller, and the TEXTOR team for their impeccable technical assistance. Merci beaucoup! Danke schön!

\section{References}

[1] Garin P. and the Tore Supra Team 1998 Proc. 20th Symp. on Fusion Tech. (Marseille) vol 2, p 1709

[2] Grisolia C. and the Tore Supra Team 1999 J. Nucl. Mater 196-198 146

[3] Weynants R.R. 1995 Plasma Phys. Control. Fusion 3763

[4] Gregory B.C. et al 1994 Contributions to High-Temperature Plasma Physics ed K.H. Spatschek and J. Uhlenbusch (Akademie Verlag, 1994)

[5] Loarer T., Gunn J.P., Lachambre J.L., Gravier E., Boucher C., Finken K.H., Lehnen M., Mank G., Jachmich S., Van Goubergen H. and Van Oost G. 2001 Czechoslovak J. Phys. 511021

[6] Van Schoor M. 1998 Modeling of biasing in tokamaks $P h D$ Thesis University of Antwerp

[7] Tendler M. and Rozhansky V. 1990 Comments Plasma Phys. Control. Fusion 13191

[8] Chankin A.V. 1997 J. Nucl. Mater. 241-243 199

[9] Staebler G.M. 1989 Nucl. Fusion 31729

[10] Hutchinson I.H. 1987 Phys. Fluids 303777

[11] Lachambre J.L. et al 1997 Plasma Phys. Control. Fusion 39 1997

[12] Van Goubergen H. 2000 PhD Thesis Universiteit Gent

[13] Van Goubergen H. et al 1999 Plasma Phys. Control. Fusion 41 L17

[14] Gunn J.P. 1998 Czechoslovak J. Phys. 48293

[15] Dyabilin K., Hron M., Stöckel J. and Žáček F. 2002 Contrib. Plasma Phys. 4299

[16] Gunn J.P. et al 2001 Phys. Plasmas 81995

[17] Gunn J.P., Stöckel J., Adámek J., Duran I., Horácek J., Hron M., Jakubka K., Kryška L., Žáček F. and Van Oost G. 2001 Czechoslovak J. Phys. 511001

[18] Van Oost G., Gunn J.P., Melnikov A., Stöckel J. and Tendler M. 2001 The role of radial electric fields in edge transport barriers in tokamaks Proc. 4th Symp. on Current Trends in International Fusion Research (Canada: NRC Research Press) 
[19] Stangeby P.C. 2000 The Plasma Boundary of Magnetic Fusion Devices (London: Institute of Physics Publishing)

[20] Chankin A.V. and Stangeby P.C. 1994 Plasma Phys. Control. Fusion 361485

[21] Chodura R. 1982 Phys. Fluids 251628

[22] Hutchinson I.H. 1996 Phys. Plasmas 36

[23] Cohen R.H. and Ryutov D. 1999 Phys. Plasmas 61995

[24] Hutchinson I.H. 1991 Phys. Fluids B 3847

[25] Hutchinson I.H. 1988 Phys. Rev. A 374358

[26] Rozhansky V. and Tendler M. 1994 Phys. Plasmas 12711

[27] Décoste R. et al 1993 Improved divertor retention and pumping with neutralising plate biasing on TdeV 20th Eur. Conf. on
Controlled Fusion and Plasma Physics (Lisbon) vol 17C part I (Petit-Lancy: European Physical Society) p 295

[28] Tsitrone E., Chappuis P., Chatelier M., Faisse F., Grosman A. and Reiter D. 1999 J. Nucl. Mater. 266-269 963

[29] Doerner R.P. et al . 1994 Nucl. Fusion 34975

[30] Mank G. et al . 2000 Investigation of particle flows by limiter biasing on TEXTOR-94 Proc. 10th Int. Congress on Plasma Physics (ICPP) vol 3, p 760

[31] MacLatchy C.S., Boucher C., Poirier D.A. and Gunn J.P. 1992 Rev. Sci. Instrum. 633923

[32] Gerhauser H., Mank G., Zagórski R., Loarer T., Gunn J.P. and Boucher C. 2001 Czechoslovak J. Phys. 501033 